\title{
Comparative in silico analyses of proteins involved in serum resistance as promising vaccine candidates against Acinetobacter baumannii
}

\author{
Soltani Shirazi A ${ }^{1}$, Azizi $\mathbf{O}^{2}$, Bolourchi $\mathbf{N}^{1}$, Aghamohammad Sh$^{1}$, Badmasti $\mathbf{F}^{1^{*}}$ \\ ${ }^{1}$ Department of Bacteriology, Pasteur Institute of Iran, Tehran, Iran. \\ ${ }^{2}$ Department of Basic Sciences, Torbat Heydariyeh University of Medical Sciences, Torbat Heydariyeh, Iran.
}

\begin{abstract}
Introduction: Acinetobacter baumannii as a Gram-negative coccobacillus has become a major cause of hospital-acquired infections. The virulence factors involved in serum resistance are important targets in the development of an effective vaccine against this pathogen. Our aim in this project was in silico analyses of A. baumannii proteins involved in serum resistance which could potentially be used as efficient vaccines. Methods: Based on computational procedures, we evaluated all A. baumannii proteins involved in serum resistance, namely AbOmpA, PKF, PLD, PBP 7/8, CipA and Tuf SurA1, as vaccine candidates. Subcellular localization, sequence conservation, domain prediction and 3D modelings were analyzed by online tools. Moreover, the prevalence of serum resistance factors in 5 strains of A. baumannii was characterized. The MHC-binding sites of class I and II were detected. Linear and conformational B cell epitopes were analyzed by 2 prediction servers. Results: The MetaLocGramN server showed that AbOmpA, PKF, PBP7/8, phospholipase D, CipA, Tuf and SurA1 were outer membrane protein (56.32\%), extracellular protein (58.74\%), extracellular protein $(52.59 \%)$, cytoplasmic protein $(45.08 \%)$, extracellular protein $(53.8 \%)$, Cytoplasmic protein $(96.36 \%)$ and extracellular protein (58.23\%), respectively. The OMD of AbOmpA, PKF, PBP7/8 and phospholipase D, CipA, Tuf and SurA1 were $0.060,0.076,0.08,0.101,0.09,0.06$ and 0.103 , respectively. The numbers of immunogenic linear and conformational epitopes with high score $(\mathrm{P} \geq 0.6)$, extracted from beta-barrel of AbOmpA were 6 and 4; whereas these values for PKF were 10 and 4 , respectively. Conclusion: The in silico analyses and reverse vaccinology criteria showed that AbOmpA and PKF had better attributes as vaccine targets and they could be considered as promising vaccine candidates against $A$. baumannii.
\end{abstract}

KEYWORDS: Acinetobacter baumannii, in silico analysis, serum resistance factors.

\section{INTRODUCTION}

Acinetobacter baumannii is a Gram-negative coccobacillus and a major causative agent of nosocomial infections. Infections caused by this bacterium could be resulted in bacteremia, urinary tract infections and surgical site infections, especially in the intensive care units [1]. Resistance genes could be easily transferred to this organism which can lead to therapeutic failures with respect to the infection [2]. In recent years, outbreaks of multidrug-resistant (MDR) strains of this bacterium in hospitals have caused worldwide health crises [1]. Therefore, new approaches based on immunological strategies such as active and passive immunizations have been developed to prevent or treat infections caused by the MDR strains.

Sepsis caused by A. baumannii could cause high mortality [3]. The virulence factors involved in serum resistance are important targets in development of an effective vaccine. OmpA of A. baumannii (AbOmpA) is one of the most important virulence factors which is involved in adherence and

\footnotetext{
*Corresponding Author: Farzad Badmasti, Department of Bacteriology, Pasteur Institute of Iran, Tehran, Iran.

Email: fbadmasti2008@gmail.com

Tel/Fax: (+98) 2166405535
}

biofilm formation by the bacterium [4]. Furthermore, new factors such as serine protease PKF, involved in serum resistance have been recently characterized. PKF is associated with resistance to complement system and suppresses the biofilm formation [5]. A study based on transposon mutant library has shown that disruption of $A$. baumannii phospholipase D (PLD) could result in reduction of serum resistance [6]. Moreover, it has been shown that a transposon mutant in an A. baumannii gene for penicillin binding protein $7 / 8$ (PBP 7/8) could lead to reduction in the virulence in a rat model of pneumonia as well as reduced serum resistance capability [7]. Meanwhile, novel serum resistance factors, including CipA as a plasminogen binding and complement inhibitory protein, elongation factor Tuf and surface antigen protein 1 (SurA1), have been characterized [8-10].

Ideally, a vaccine candidate protein should play critical roles in pathogenesis of a bacterium and be conserved amongst all the related strains. Moreover, it should be exposed to extracellular spaces to be easily presented to the immune system. Having one or more immunogenic epitopes that elicit a protective immune response in humans or animal models is another important feature for a protein to be an efficient vaccine candidate [11]. In 
this regard, here we used bioinformatics tools to assess A. baumannii proteins involved in serum resistance in order to define potentially effective vaccine targets for this pathogen.

\section{MATERIALS and METHODS}

\section{Comparative analyses}

All serum resistance proteins were retrieved in FASTA format from Uniprot database <http://www.uniprot.org/> [12]. The subcellular localizations of the proteins were detected by MetaLocGramN <http://genesilico.pl/MetaLocGramN/>; a meta-server for subcellular localization and prediction of Gramnegative proteins (i.e. proteins which are extracted from Gramnegative bacteria) [13]. All domains of the proteins were identified in NCBI CD-search <http://www.ncbi.nlm.nih.gov/Structure/cdd/wrpsb.cgi> domain prediction tool [14]. VICMpred was applied to predict the role of the protein in bacteria <http://www.imtech.res.in/raghava/vicmpred/>; which is a Support Vector Machine (SVM)-based method using amino acid patterns and composition to predict functional proteins of Gram-negative bacteria [15]. The overall antigenicity of proteins was evaluated in VaxiJen <http://www.ddgpharmfac.net/vaxijen/VaxiJen/VaxiJen.html > [16]. The adhesion probability, trans-membrane helices and similarity to eukaryotic proteins were checked in Vaxitop server <http://www.violinet.org/vaxign/vaxitop/index.php> [17].

Prevalence of serum resistance factors in A. baumannii strains

The prevalence of serum resistance factors in 5 strains of A. baumannii was characterized by bacterial genome BLAST tool in ENSEMBL server <http://bacteria.ensembl.org/Multi/Tools/Blast> [18]. A. baumannii AB0057 (ST-1, international clone -I), ACICU (ST-2, international clone-II), ATCC 17978 (Non-international clone), MDR-TJ (as a multi-drug resistant), D1279779 (agent of community acquired infection) and SDF (non-pathogenic) strains were considered in this analysis. These 5 strains covered the basic genome structure of A. baumannii, as previously reported [37]. The whole genomes of these strains have been sequenced previously and deposited in genome database.

Sequence conservation analysis

The ConSurf < http://consurf.tau.ac.il/>, a server for estimating the evolutionary conservation of amino/nucleic acid positions in a protein/DNA/RNA molecule, based on the phylogenetic relations between homologous sequences [19], was used for identification of the conserved regions in the mentioned proteins. Moreover, we computed the overall mean distance (OMD) of 100 non-redundant protein sequences of each serum resistance factors by MEGA v6.0 software [20]. This value represents $1 /$ conservation.

\section{MHC binding sites prediction}

The MHC binding sites of class I and II were detected by Vaxitop server <http://www.violinet.org/vaxign/vaxitop/index.p $\mathrm{hp}>$. In this analysis, all human MHC alleles with any lengths of MHC binding sites were considered. The probability $(P-$ value) of all binding sites was less than 0.05 . The number of $\mathrm{MCH}$ binding sites (separately for MHC-I and MHC-II) were divided to number of whole amino acids of proteins and were reported as a ratio in Table 1.

\section{D molecular modeling}

The secondary structures of the proteins were predicted by Jpred server < http://www.compbio.dundee.ac.uk/jpred/index_u p.html>; a protein secondary structure prediction server [21]. Prediction of 3D molecular modeling of promising targets was done by local meta-threading server LOMETS <http://zhanglab.ccmb.med.umich.edu/LOMETS/>; an online web service to predict the structure of the protein which collects consensus contact predictions from multiple threading templates [22]. The best models with the highest score were selected. The predicted models were subjected to further refinement in ModRefiner server <http://zhanglab.ccmb.med.umich.edu/ModRefiner/> and energy minimization of predicted models were performed at high-resolution protein structure refinement web server [23].

Linear and conformational B cell epitopes prediction Linear and conformational B cell epitopes and putative antigenic determinants were analyzed by ElliPro Prediction server [24]; a web-tool that implements Thornton's method and allows the prediction and visualization of antibody epitopes in a given protein sequence or structure together with a residue clustering algorithm <http://tools.immuneepitope.org/tools/Elli Pro/iedb_input $>$. Based on the 3D structure of a protein antigen, ElliPro predicts linear and discontinuous antibody epitopes by homology modeling. Moreover, the conformational B cell epitope was obtained by Discotope server <http://www.cbs.dtu.dk/services/DiscoTope/> for comparison with Ellipro results.

\section{RESULTS}

Subcellular localization and sequence conservation. MetaLocGramN server showed AbOmpA, PKF, PBP7/8, phospholipase D, CipA, Tuf and SurA1 were outer membrane protein $(56.32 \%)$, extracellular protein $(58.74 \%)$, extracellular protein $(52.59 \%)$, cytoplasmic protein $(45.08 \%)$, extracellular protein (53.8\%), Cytoplasmic protein $(96.36 \%)$ and extracellular protein $(58.23 \%)$, respectively. These results are represented in Table 1. The OMD of AbOmpA, PKF, PBP7/8 and phospholipase D, CipA, Tuf and SurA1 were 0.060, 0.076, $0.08,0.101,0.09,0.06$ and 0.103 , respectively.

\section{Domain prediction}

Domain prediction showed AbOmpA had two independent domains as follows: beta-barrel domain which could be found wildly in outer membrane proteins. This domain assumes a membrane-bound beta-barrel fold C-terminal domain AbOmpA; OmpA-like domains have been shown to noncovalently associated with peptidoglycan [25]. The secreted serine protease PKF had trypsin-like serine protease activity (Peptidase S1). Moreover, it had two PDZ domain of trypsinlike serine proteases, like DegP/HtrA, which are oligomeric proteins involved in chaperone function, apoptosis and heatshock response [26]. This domain could play major role in substrate recognition and/or binding to C-terminal polypeptides. Penicillin binding protein $7 / 8$ with D-alanyl-D-alanine carboxypeptidase activity had transpeptidase domain [27]. The active site of this protein has a serine residue that is conserved in all members of this family. Phospholipase D (PLD) had PLD-like domain with two repeats of catalytic domains which have been named repeat-1 and repeat-2. Phospholipid phosphodiester bonds could be hydrolyzed by PLD enzymes. This catalysis causes phosphatidic acid as well as a free polar head group. They can also catalyze trans-phosphatidylation of phospholipids to acceptor alcohols [28]. CipA protein has META domain; a small domain family found in proteins of 
Table 1. The summarized information of comparative in silico analyses of proteins involved in serum resistance of A. baumannii

\begin{tabular}{|c|c|c|c|c|c|c|c|c|c|c|c|c|c|c|c|c|c|c|}
\hline Protein & GenBank ID & Length & $\begin{array}{l}\text { Domain or } \\
\text { function }\end{array}$ & $\begin{array}{c}\mathrm{AB} 00 \\
57\end{array}$ & $\begin{array}{l}\mathrm{ACI} \\
\mathrm{CU}\end{array}$ & $\begin{array}{l}\text { ATCC } \\
17978\end{array}$ & $\begin{array}{l}\text { MD } \\
\text { R-TJ }\end{array}$ & $\begin{array}{c}\text { D1279 } \\
779\end{array}$ & SDF & $\begin{array}{l}\text { MetaLocGra } \\
\mathrm{mN}^{\mathrm{a}}\end{array}$ & $\begin{array}{l}\text { Adhesion } \\
\text { Probability }\end{array}$ & $\begin{array}{l}\text { Trans- } \\
\text { membrane } \\
\text { helices }\end{array}$ & $\begin{array}{c}\text { Similar to } \\
\text { Eukaryotic } \\
\text { proteins }\end{array}$ & $\begin{array}{l}\text { VICM- } \\
\text { pred }^{\text {b }}\end{array}$ & $\begin{array}{l}\text { Signal } \\
\text { peptide }\end{array}$ & $\mathrm{OMD}^{\mathrm{d}}$ & $\begin{array}{l}\text { Protein } \\
\text { Antigen } \\
\text { icity }\end{array}$ & $\begin{array}{c}\text { No. of } \\
\text { MHC- } \\
\text { class I } \\
\text { and II } \\
\text { binding } \\
\text { sites per } \\
\text { amino } \\
\text { acid }\end{array}$ \\
\hline $\begin{array}{c}\text { OmpA of } \\
\text { Acinetobac } \\
\text { ter } \\
\text { baumannii( } \\
\text { AbOmpA) }\end{array}$ & ACICU_03089 & 356 aa & $\begin{array}{c}\text { Beta barrel } \\
\text { domain, C- } \\
\text { terminal ompA- } \\
\text { like }\end{array}$ & + & + & + & + & + & + & $\mathrm{OM}(56.32 \%)$ & 0.476 & 1 & No & $\begin{array}{c}\mathrm{CP} \\
(3.01)\end{array}$ & $\begin{array}{c}\text { SpI } \\
(13.98)\end{array}$ & 0.060 & 0.8457 & $\begin{array}{l}\text { MHC- } \\
\text { I: } 1.77 \\
\text { MHC-II: } \\
0.83\end{array}$ \\
\hline $\begin{array}{l}\text { Serine } \\
\text { proteases } \\
(\mathrm{PKF})\end{array}$ & ACICU_02801 & 458 aa & $\begin{array}{c}\text { Trypsin-like } \\
\text { cysteine/serine } \\
\text { peptidase domain, } \\
\text { PDZ domain } \\
\end{array}$ & + & + & + & + & + & + & EC $(58.74 \%)$ & 0.205 & 0 & No & $\begin{array}{c}\mathrm{VF} \\
(1.35)\end{array}$ & $\begin{array}{c}\text { SpI } \\
(8.19)\end{array}$ & 0.076 & 0.5524 & $\begin{array}{c}\text { MHC-I: } \\
1.98 \\
\text { MHC-II:: } \\
0.77 \\
\end{array}$ \\
\hline $\begin{array}{l}\text { penicillin } \\
\text { binding } \\
\text { protein } 7 / 8 \\
(\mathrm{Pbp} 7 / 8)\end{array}$ & ACICU_00260 & 340 aа & $\begin{array}{l}\text { Penicillin binding } \\
\text { protein } \\
\text { transpeptidase } \\
\text { domain (D- } \\
\text { alanyl-D-alanine } \\
\text { carboxypeptidase) }\end{array}$ & + & + & + & + & + & + & $\mathrm{EC}(52.59 \%)$ & 0.495 & 0 & No & $\begin{array}{c}\mathrm{VF} \\
(1.22)\end{array}$ & $\begin{array}{c}\mathrm{SpI} \\
(22.89)\end{array}$ & 0.08 & 0.5417 & $\begin{array}{c}\text { MHC-I: } \\
1.92 \\
\\
\text { MHC-II: } \\
0.73 \\
\end{array}$ \\
\hline $\begin{array}{l}\text { Phospholip } \\
\text { ase D-like } \\
\text { proteins } \\
\text { (PLD) } \\
\end{array}$ & ACICU_03141 & 487 aа & PLD-like domain & + & + & + & + & + & + & $\mathrm{CP}(45.08 \%)$ & 0.266 & 1 & No & $\begin{array}{c}\mathrm{VF} \\
(0.65)\end{array}$ & $\begin{array}{c}\text { TMH } \\
(1.15)\end{array}$ & 0.101 & 0.4507 & $\begin{array}{c}\text { MHC-I: } \\
2.17 \\
\text { MHC-II: } \\
1.06 \\
\end{array}$ \\
\hline CipA & ACICU_00989 & 369 aа & $\begin{array}{l}\text { META domain; } \\
\text { Small domain } \\
\text { family found in } \\
\text { proteins of } \\
\text { unknown } \\
\text { function. } \\
\end{array}$ & + & + & + & + & + & + & $\mathrm{EC}(53.8 \%)$ & 0.651 & 0 & No & $\begin{array}{c}\mathrm{MM} \\
(1.03)\end{array}$ & $\begin{array}{c}\text { SpII } \\
(10.87)\end{array}$ & 0.09 & 0.6646 & $\begin{array}{c}\text { MHC-I: } \\
1.87 \\
\text { MHC-II: } \\
0.91\end{array}$ \\
\hline Tuf & AB57_0914 & 396 aа & $\begin{array}{c}\text { Selenocysteine- } \\
\text { specific } \\
\text { translation } \\
\text { elongation factor } \\
\end{array}$ & + & + & + & + & + & + & CP $(96.36 \%)$ & 0.097 & 0 & Yes & $\begin{array}{c}\text { IS } \\
(4.64)\end{array}$ & $\begin{array}{l}\text { CYP } \\
(0.2)\end{array}$ & 0.06 & 0.5218 & $\begin{array}{c}\text { MHC-I: } \\
1.92 \\
\text { MHC-II: } \\
0.83 \\
\end{array}$ \\
\hline $\begin{array}{l}\text { surface } \\
\text { antigen } \\
\text { protein } 1 \\
\text { (SurA1) } \\
\end{array}$ & AB57_1613 & 105 aа & No domain & + & + & + & + & + & - & $\mathrm{EC}(58.23 \%)$ & 0.298 & 0 & No & $\begin{array}{c}\text { MM } \\
(1.50)\end{array}$ & $\begin{array}{l}\text { CYP } \\
(0.2)\end{array}$ & 0.103 & 0.6738 & $\begin{array}{c}\text { MHC-I: } \\
1.66 \\
\text { MHC-II: } \\
0.62 \\
\end{array}$ \\
\hline
\end{tabular}

a: OM, Outer membrane; EC, Extracellular; CP, Cytoplasmic

b: CP, Cellular process; VF, Virulence factor; MM, Metabolism Molecule; IS, Information and storage

c: SpI, Signal peptide type I; TMH, N-terminal transmembrane helix; CYP, Cytoplasmic

d: OMD, overall mean distance. 
Analysis of proteins based on reverse vaccinology criteria All proteins (except SurA1) were highly prevalent in selected strains. All proteins (except Tuf) were not similar to eukaryote proteins. All proteins (except PLD) had $P>0.5$ overall antigenicity score. The VICMpred and signal peptide predictions showed all proteins (except CipA) were putative virulence factors and maybe directly or indirectly involved in bacterial pathogenesis (see Table 1). Taken together, our analyses showed AbOmpA and PKF are two best vaccine targets. Therefore, the following sections and other analyses were performed just on these two proteins.

3D modeling and B cell epitope predictions

A

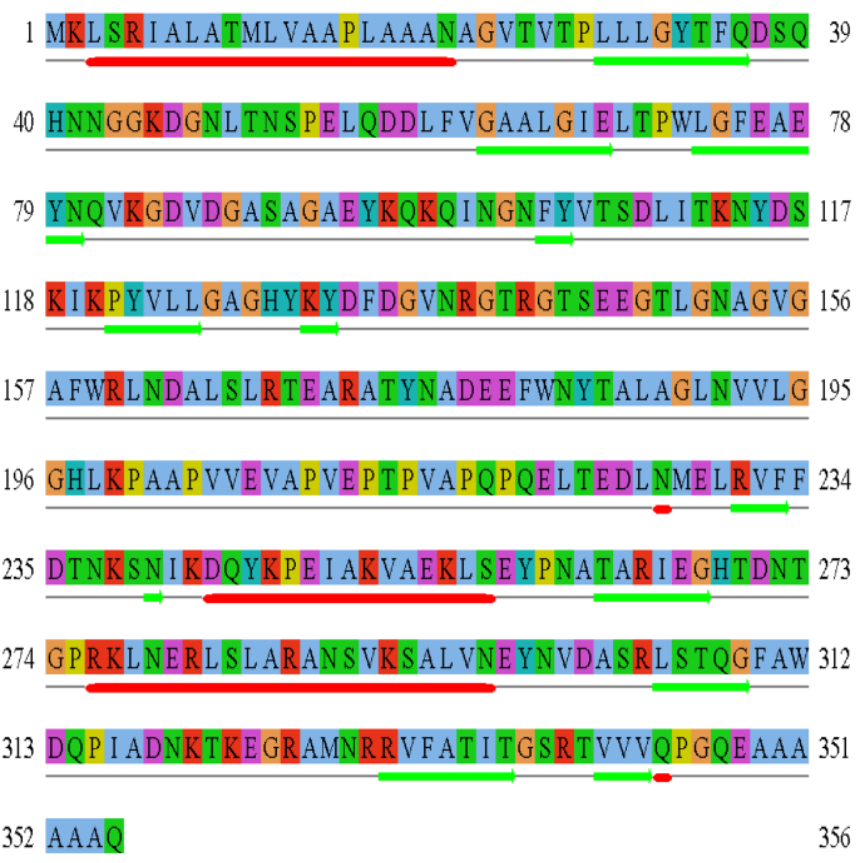

C

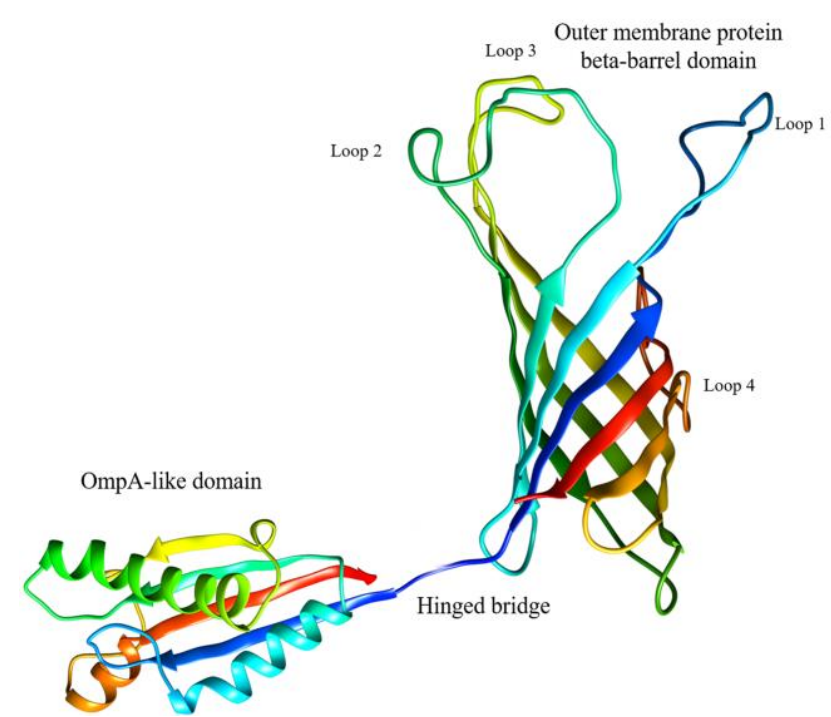

These following procedures were performed only for AbOmpA and PKF, as promising vaccine targets. Data have shown that the beta-barrel domain of AbOmpA is similar to beta-barrel domain of Klebsiella pneumoniae OmpA (PDB accession number is $2 \mathrm{~K} 0 \mathrm{~L}$ ) [29]. This domain has 4 extracellular loops. The PKF protein is homolog of DegP (PDB accession number 2ZLE). The heat-shock protein DegP is a protein quality control factor which plays different roles in regulating protease, eliminating misfolded proteins and in the biogenesis of outermembrane proteins in Escherichia coli and Legionella pneumophila [30]. The secondary structures and hypothetical 3D model of AbOmpA and PKF protein are shown in Fig. 1.

B

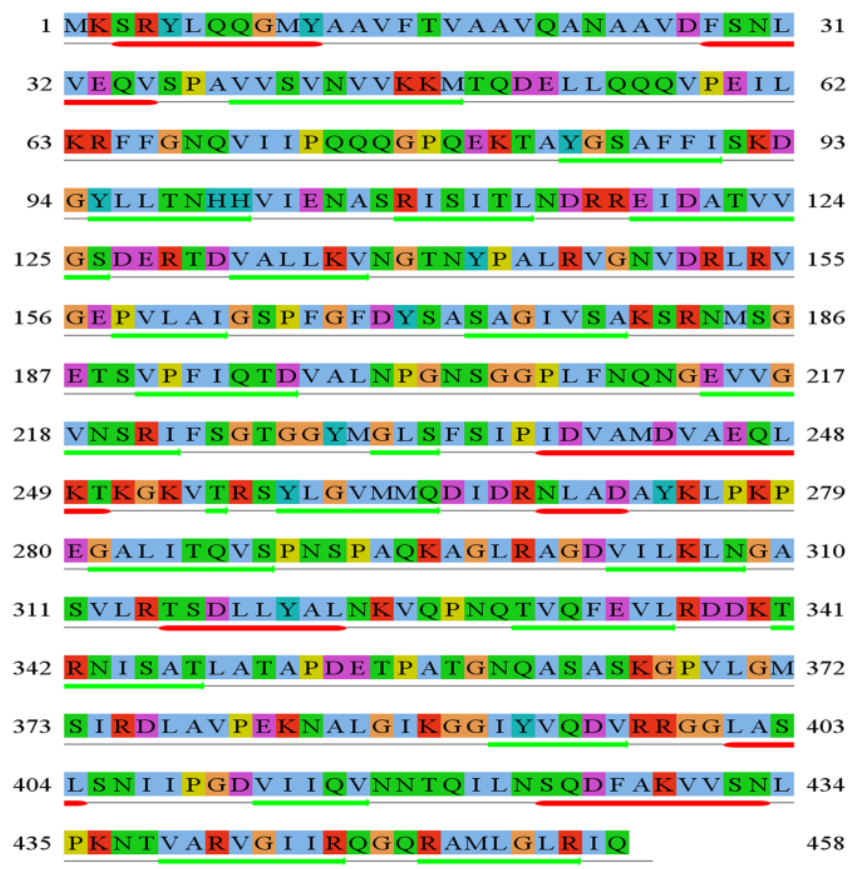

D

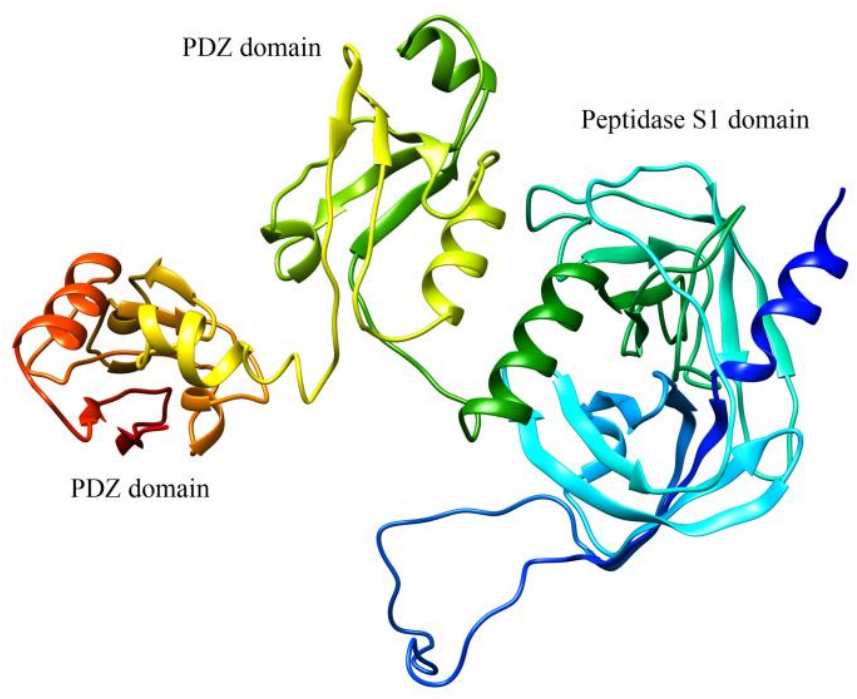

Fig. 1. The secondary structures of AbOmpA (1A) and PKF (1B). Amino acid sequences have shown in ClustalX format and the secondary structure represented by Jnetpred (red ribbons are alpha helix; green ribbons are beta strand). The hypothetical model of AbOmpA protein (1C). The N-terminal domain (beta-barrel domain) folds in outer membrane of bacterium which is connected to C-terminal domain by glycine-proline-rich linker. The C-terminal domain (globular domain) interacts with peptidoglycan. The refined model of secreted serine protease PKF (1D). It seems that PKF can be polymerized and constructs a compartment. 
We analyzed two proteins (beta barrel domain of AbOmpA and full length PKF protein) by ConSurf tool. These data showed conservation and protrusion of every amino acid, one by one,

A

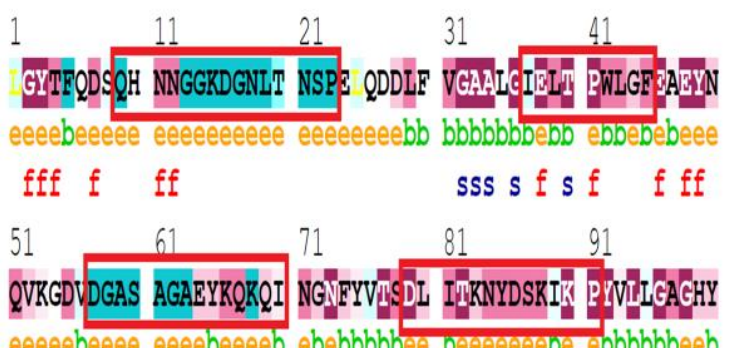

ceeeebeeee reeebeeeeb ebebbbbbee beececedoe abbbbbeeb

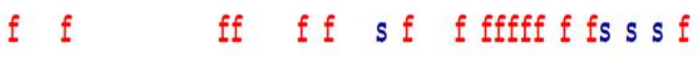
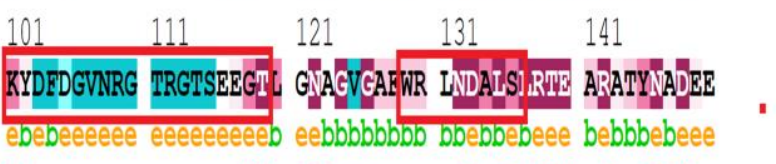

$f \quad$ ff $f S S$ sf sisfif $f$ if

$151 \quad 161$

\section{FWNMALAG N}

bbebbbbeee e

sf $s$ fff $f$

Legend:

The conservation scale:

122344567789

Variable Average Conserved

e - An exposed residue according to the neural-network algorithm.

b - A buried residue according to the neural-network algorithm.

f - A predicted functional residue (highly conserved and exposed).

s- A predicted structural residue (highly conserved and buried).

- Insufficient data - the calculation for this site was performed on less than $10 \%$ of the sequences. that could be used in evaluation of linear B cell epitopes. The results have been represented in Fig. 2.

B

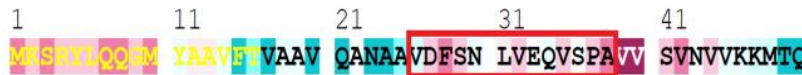
eeeeebeebb bbbbbbbbee eeeeeebee bbeebeeebb bbebeeeee $f \mathrm{f}$ ff fss

$\begin{array}{lllll}51 & 61 & 71 & 81 & 91\end{array}$ DELLQQQVPE ILKRFFGNQV IIPQQQGPQE KTAY JSAEFI SKDEYLLTNH eeeeeeeee bbeebbeee eeeeeeee eeeeebbbb beebbbbbee ffss $\quad s$ sf

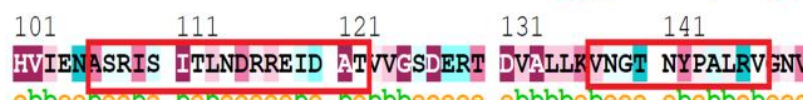
ebbeebee Debeecelo Debbeeee ebbbbeee ebbbebee fs $s$ f $s$ s $f$ if $s$

$\begin{array}{lllll}151 & 161 & 171 & 181 & 191\end{array}$

DRLRVGEPVI AIGS ?FGFDY SASAGI YSAR SRMMSGETSV PFIQIDVALN eebebbebbb bbbbbbebee ebbebbbbe eeebeee be ebbebebbee sf $\quad$ sss $f \quad f$ s ss ss $f \quad$ sfsfs $f f$ $\begin{array}{lllll}201 & 211 & 221 & 231 & 241\end{array}$

PGNSGGPLFN QNGEVVGVNS RIFSGTGGYM GLSESIPIDV AMDVAEQIKT eeeeee bbb bebebbbbeb bbebeeeee b.bbbbbbeb beebbeebee ffffffes $s \quad s$ S $f$ s $f$ f s ssss

$\begin{array}{lllll}251 & 261 & 271 & 281 & 291\end{array}$

KGKVTRSYLC VMMQDIDRNL ADAYKLPKPE GALITQVSPN SPÄQKAGLRA eeebeebbbb bebeebeeeb eeeebeee abbbebeee eebeeeebee $f \quad f \quad s \quad f \quad f \quad$ f $s$ ff

$3013311 \quad 321 \quad 331 \quad 341$

GDVILKLNGA SVLRTSDLLY ALNKVQPNQT VQFEVLRDDK TRNISATLAT bebbbebeee ebeeeeebee ebeeeeeee bebebbeee eeebebee f

$\begin{array}{lllll}351 & 361 & 371 & 381 & 391 \\ \text { APDETPATGN } & \text { QASASKGPVI GMSIRDLAVP EKNALGIKGG IY VQDVRRGG }\end{array}$ eeeeeeeee eeeeeeeeb ebebee eeeebeeee brbeebeee

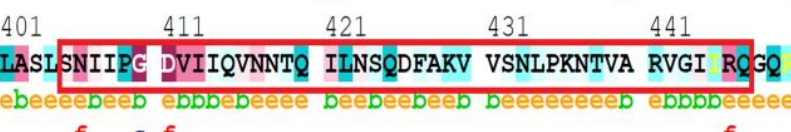
$f \quad s f$ 451

AM GLRI

bbbbbeee

Fig. 2. Predictions of amino acid conservation and linear B cell epitopes of beta barrel domain of AbOmpA (2A) and full length PKF protein (2B) showed major linear B cell epitopes in beta barrel domain of AbOmpA and full length PKF protein (red rectangles). However, the beta barrel domain of AbOmpA had 3 conserved epitopes while PKF had 4 conserved epitopes.

Based on Ellipro results, the numbers of immunogenic linear and conformational epitopes with high score $(P \geq 0.6)$, extracted from beta-barrel of AbOmpA were 6 and 4. Whereas,
PKF had 10 and 4, respectively. The DiscoTope results also matched to Ellipro results (Fig. 3). 
A
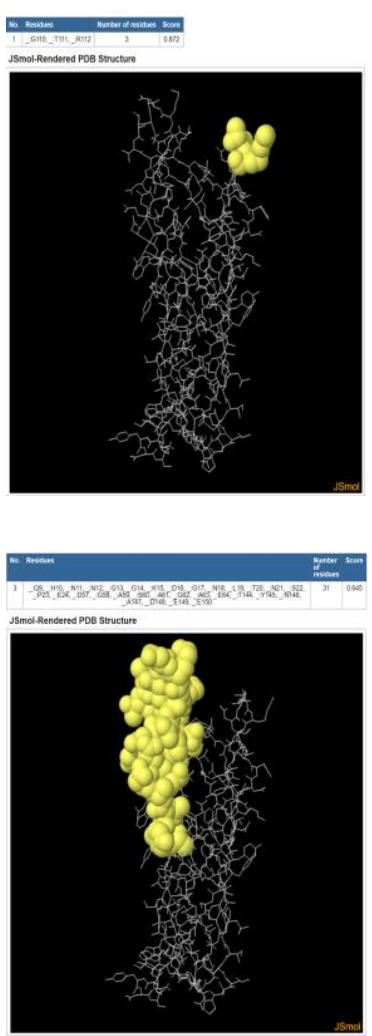

C
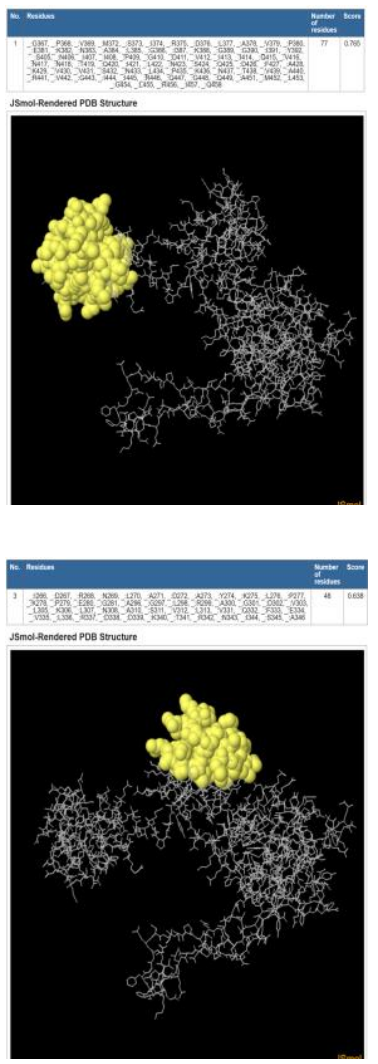
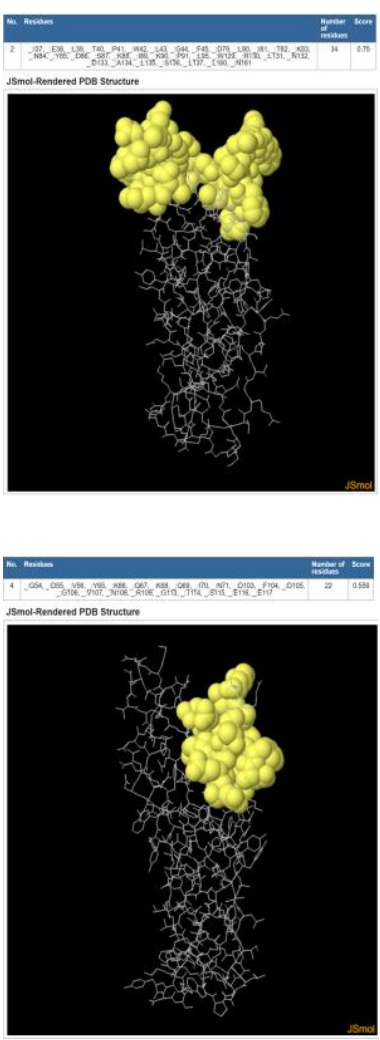

B

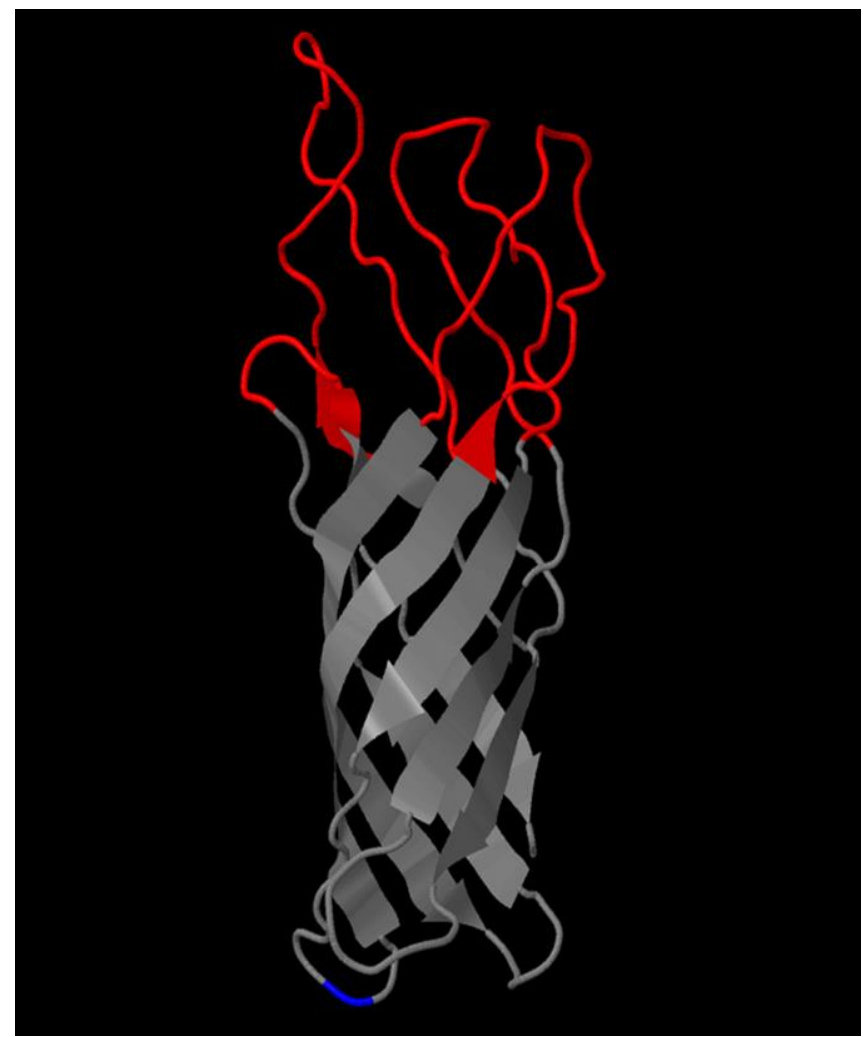

D
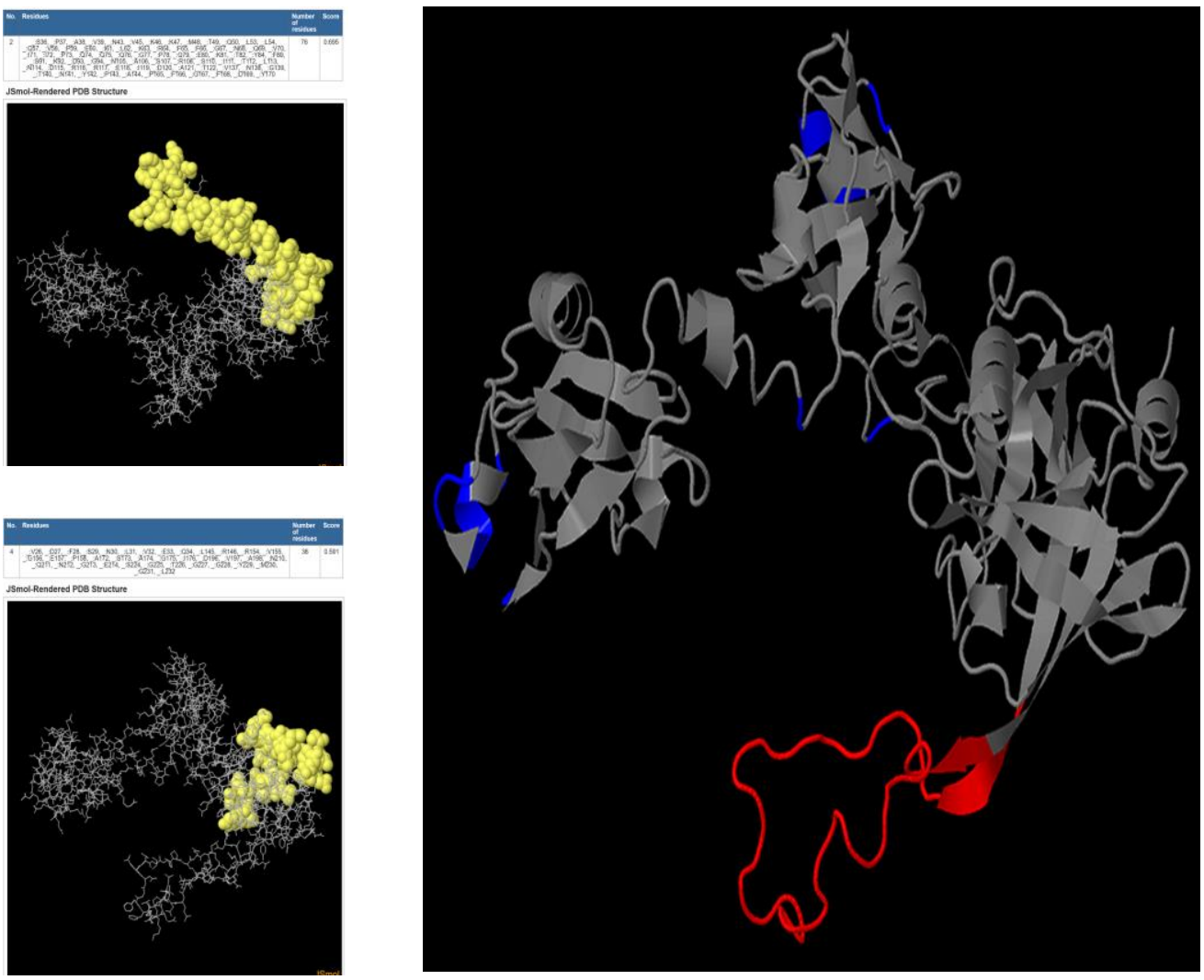

Fig. 3. The conformational B cell epitopes of beta-barrel domain of AbOmpA and full length PKF protein by Ellipro and DiscoTope. Data showed that the major epitopes in beta-barrel domain of AbOmpA were located on 4 extracellular loops (see 3A and 3B). Moreover, our analysis showed that the conformational epitopes of PKF have been distributed on all domains of this protein (see 3C and 3D). Ellipro results show the amino acid positions of each epitope in yellow color. DiscoTope results show the conformational epitopes $(\operatorname{Red}=$ high score, Blue $=$ low score $)$. 


\section{DISCUSSION}

A previous investigation has proposed several proteins as novel vaccine candidates against $A$. baumannii where proteomic analysis and MALDI-TOF/TOF of outer membrane proteins from $A$. baumannii have revealed 6 immune-reactive proteins, namely Omp34kDa, OmpA, OprB-like, OprC, OXA-23, and iron regulated outer membrane proteins. Remarkably, these proteins are extensively abundant on the bacterial surface and are involved in virulence, growth and antimicrobial resistance [31]. Moreover, reverse vaccinology and in vitro proteomic approaches have identified 42 antigens that could be used as potential vaccine targets against MDR $A$. baumannii. These proteins have been found mostly in secretome and/or outer membrane vesicles (OMV), as extracellular or surface-exposed proteins [32]. Bioinformatics tools are now a standard methodology in vaccine research to discover effective candidate proteins and epitopes which could potentially induce proper immune responses [33]. Moreover, reverse vaccinology has a great potential for identification of novel targets for such purposes. For instance, one study has identified 35 outer membrane or extracellular adhesins, as promising A. baumannii vaccine targets. [34].

Among proteins of interest from A. baumannii, AbOmpA has recently been shown to be involved in serum resistance. It has been shown that AbOmpA would prevent the killing of the bacterium by the complement system, since it could interact with soluble inhibitors of the alternative complement pathway [35]. AbOmpA attaches to Factor $\mathrm{H}$ (inhibitor of alternative complement) and protects A. baumannii form MAC activation [35]. Moreover, PKF as a secreted serine protease may hydrolyze the complement factor that gives rise to the serum resistance [5]. However, the serum resistance mechanisms of PBP7/8 and PLD are not clear so far. Comparative in silico analyses have shown that both AbOmpA and PKF have satisfied characteristics to be considered as vaccine candidates against $A$. baumannii. These proteins have clear roles in bacterial pathogenesis and are more conserved in comparison with other mentioned proteins. They are also surface exposed or secreted proteins, have many MHC binding sites and contain strong linear and conformational B cell epitopes.

Although the role of PBP-7/8 in Gram-negative bacteria is not totally clear, it is known to be an accessory enzyme involved in modulation of cell morphology and separation of daughter cells, without being essential in normal cell elongation. It may also take part in serum resistance, indirectly [27]. In our study this protein had a promising prospect as vaccine target but its pathogenic roles as virulence factor is not clear. Although experimental studies have confirmed that PLD is an A. baumannii virulence factor, this protein is not conserved at all (OMD was 0.101). Moreover, it is localized in cytoplasmic fluid.

The in silico analyses of AbOmpA showed this protein forms two independent domain structures; namely, N-terminal and Cterminal domains. The $\mathrm{N}$-terminal forms an 8 strand beta-barrel channel which has 4 loops, exposed to the extracellular spaces. A soluble and stable globular domain in C-terminal of the protein interacts with the peptidoglycan layer in the periplasmic space and it is required for the integrity of the cell [36]. Multiple sequence alignments of beta-barrel domain showed L4 was conserved while L1, L3, and L2 had more amino acid changes [29]. The extracellular loops are functional and dynamic regions of this protein which could be involved in adherence, invasiveness, biofilm formation, apoptosis, immune stimulation, iron metabolism and serum resistance.

The ConSurf plot and OMD showed AbOmpA protein is totally a conserved protein. The experimental studies have also shown that it has crucial roles in pathogenesis of A. baumannii. Linear $\mathrm{B}$ cell epitope predictions reveled that these epitopes are located on the extracellular loops (see Fig. 2). Although the ConSurf plot showed $\mathrm{N}$ - and C-terminals of PKF protein are variable, the active site of this enzyme was conserved (OMD was 0.076). $\mathrm{PKF}$ factor as a secreted protein (MetaLocGramN score was $58.74 \%$ ) had more linear and conformational B cell epitopes in comparison with beta-barrel domain of AbOmpA. Moreover, epitope prediction results of $\mathrm{B}$ and $\mathrm{T}$ cells suggest that the extracellular loops of beta-barrel domain had prominent characteristics as immunogenic epitopes which could stimulate both humoral and cellular immunities. It is envisaged that in principle, such extracellular loops could also be used for delivering short immunogenic epitopes of other pathogenic bacteria as vaccine targets, either alone or in fusion forms, to elicit strong responses against $A$. baumannii.

\section{ACKNOWLEDGEMENT}

The authors would like to thank the personnel in Department of Bacteriology of Pasture Institute of Iran for their help. This research was supported by Pasture Institute of Iran under grant number 911, donated to Farzad Badmasti.

\section{CONFLICT OF INTEREST}

The authors declare that they have no conflict of interest.

\section{REFERENCES}

1. Dijkshoorn L, Nemec A, Seifert H. An increasing threat in hospitals: multidrug-resistant Acinetobacter baumannii. NAT rev microbiol. 2007;5(12):939-51. doi:10.1038/nrmicro1789.

2. Gordon NC, Wareham DW. Multidrug-resistant Acinetobacter baumannii: mechanisms of virulence and resistance. Int $\mathrm{J}$ Antimicrob Agents . 2010;35(3):219-26. doi:10.1016/j.ijantimicag.2009.10.024.

3. Eliopoulos GM, Maragakis LL, Perl TM. Acinetobacter baumannii: epidemiology, antimicrobial resistance, and treatment options Clin Infect Dis. . 2008;46(8):1254-63. doi:10.1086/529198.

4. Mortensen BL, Skaar EP. Host-microbe interactions that shape the pathogenesis of Acinetobacter baumannii infection. Cell Microbiol. 2012;14(9):1336-44. doi:10.1111/j.1462-5822.2012.01817.x.

5. King LB, Pangburn MK, McDaniel LS. Serine protease PKF of Acinetobacter baumannii results in serum resistance and suppression of biofilm formation. J Infect Dis. 2013:jis939. doi: 10.1093/infdis/jis939.

6. Jacobs AC, Hood I, Boyd KL, Olson PD, Morrison JM, Carson S et al. Inactivation of phospholipase $\mathrm{D}$ diminishes Acinetobacter baumannii pathogenesis. Infection and immunity. 2010;78(5):1952-62. doi: 10.1128/IAI.00889-09.

7. Russo TA, MacDonald U, Beanan JM, Olson R, MacDonald IJ, Sauberan SL et al. Penicillin-binding protein $7 / 8$ contributes to the survival of Acinetobacter baumannii in vitro and in vivo. $J$ Infect Dis. 2009;199(4):513-21. doi: 10.1086/596317.

8. Koenigs A, Stahl J, Averhoff B, Göttig S, Wichelhaus TA, Wallich R et al. CipA of Acinetobacter baumannii is a novel plasminogen binding and complement inhibitory protein. The J Infect Dis. 2015;213(9):1388-99. doi: 10.1093/infdis/jiv601.

9. Koenigs A, Zipfel PF, Kraiczy P. Translation elongation factor Tuf of Acinetobacter baumannii is a plasminogen-binding protein. PloS one. 2015;10(7):e0134418. doi: 10.1371/journal.pone.0134418.

10. Liu D, Liu Z-S, Hu P, Cai L, Fu B-Q, Li Y-S et al. Characterization of surface antigen protein 1 (SurA1) from Acinetobacter baumannii and its role in virulence and fitness. Vet Microbiol. 2016;186:126-38. doi: 10.1016/j.vetmic.2016.02.018. 
11. Moxon R, Rappuoli R. Bacterial pathogen genomics and vaccines. $\mathrm{Br}$ Med Bull. 2002;62(1):45-58. doi.org/10.1093/bmb/62.1.45.

12. Apweiler R, Bairoch A, Wu CH, Barker WC, Boeckmann B, Ferro S et al. UniProt: the universal protein knowledgebase. Nucleic Acids Res. 2004;32(suppl 1):D115-D9. https://doi.org/10.1093/nar/gkw1099.

13. Magnus M, Pawlowski M, Bujnicki JM. MetaLocGramN: a metapredictor of protein subcellular localization for Gram-negative bacteria. Biochim Biophys Acta. 2012;1824(12):1425-33. doi: 10.1016/j.bbapap.2012.05.018.

14. Marchler-Bauer A, Lu S, Anderson JB, Chitsaz F, Derbyshire MK, DeWeese-Scott $\mathrm{C}$ et al. CDD: a Conserved Domain Database for the functional annotation of proteins. Nucleic Acids Res. 2011;39(suppl 1):D225-D9. doi: 10.1093/nar/gkq1189.

15. Saha S, Raghava G. VICMpred: an SVM-based method for the prediction of functional proteins of Gram-negative bacteria using amino acid patterns and composition. GPB. 2006;4(1):42-7. doi: 10.1016/S16720229(06)60015-6.

16. Doytchinova IA, Flower DR. VaxiJen: a server for prediction of protective antigens, tumour antigens and subunit vaccines. BMC bioinformatics. 2007;8(1):4. doi.org/10.1186/1471-2105-8-4.

17. He Y, Xiang Z, Mobley HL. Vaxign: the first web-based vaccine design program for reverse vaccinology and applications for vaccine development. Biomed Res Int. 2010;2010, doi: 10.1155/2010/297505.

18. Ruffier M, Kähäri A, Komorowska M, Keenan S, Laird M, Longden I et al. Ensembl core software resources: storage and programmatic access for DNA sequence and genome annotation. Database. 2017;2017. doi 10.1093/database/bax020.

19. Glaser F, Pupko T, Paz I, Bell RE, Bechor-Shental D, Martz E et al. ConSurf: identification of functional regions in proteins by surfacemapping of phylogenetic information. Bioinformatics. 2003;19(1):163-4. doi: 10.1093/bioinformatics/19.1.163.

20. Tamura K, Stecher G, Peterson D, Filipski A, Kumar S. MEGA6: molecular evolutionary genetics analysis version 6.0. Molecular biology and evolution. 2013;30(12):2725-9. DOI: 10.1093/molbev/mst197.

21. Drozdetskiy A, Cole C, Procter J, Barton GJ. JPred4: a protein secondary structure prediction server. Nucleic acids res. 2015;43(W1):W389-W94. doi.org/10.1093/nar/gkv332.

22. Wu S, Zhang Y. LOMETS: a local meta-threading-server for protein structure prediction. Nucleic acids res. 2007;35(10):3375-82. doi: $10.1093 /$ nar/gkm251.

23. Xu D, Zhang Y. Improving the physical realism and structural accuracy of protein models by a two-step atomic-level energy minimization. Biophysical J. 2011;101(10):2525-34. doi: 10.1016/j.bpj.2011.10.024.

24. Ponomarenko J, Bui H-H, Li W, Fusseder N, Bourne PE, Sette A et al. ElliPro: a new structure-based tool for the prediction of antibody epitopes. BMC bioinformatics. 2008;9(1):514. doi: 10.1186/1471-2105-9-514.
25. Park JS, Lee WC, Yeo KJ, Ryu K-S, Kumarasiri M, Hesek D et al. Mechanism of anchoring of OmpA protein to the cell wall peptidoglycan of the gram-negative bacterial outer membrane. The FASEB J. 2012;26(1):219-28. doi. 10.1096/fj.11-188425.

26. Wessler S, Schneider G, Backert S. Bacterial serine protease HtrA as a promising new target for antimicrobial therapy Cell Commun Signal.. 2017;15(1):4. doi:10.1186/s12964-017-0162-5.

27. Sauvage E, Kerff F, Terrak M, Ayala JA, Charlier P. The penicillinbinding proteins: structure and role in peptidoglycan biosynthesis. FEMS Microbiol Rev. 2008;32(2):234-58. doi: 10.1111/j.1574-6976.2008.00105.x. 28. Selvy PE, Lavieri RR, Lindsley CW, Brown HA. Phospholipase D: enzymology, functionality, and chemical modulation. Chem. Rev. 2011;111(10):6064-119. doi: 10.1021/cr200296t.

29. Badmasti F, Siadat S, Bouzari S, Nasiri O, Nemati H, Shahcheraghi F. Molecular analysis of AbOmpA type-1 as immunogenic target for therapeutic interventions against MDR Acinetobacter baumannii infection. Vaccine Res. 2015;2(3):9-18. doi:10.18869/acadpub.vacres.2.3.9.

30. Krojer T, Sawa J, Schäfer E, Saibil HR, Ehrmann M, Clausen T. Structural basis for the regulated protease and chaperone function of DegP. Nature. 2008;453(7197):885-90. doi: 10.1038/nature07004.

31. Fajardo Bonin R, Chapeaurouge A, Perales J, Silva JG, do Nascimento HJ, D'Alincourt Carvalho Assef AP et al. Identification of immunogenic proteins of the bacterium Acinetobacter baumannii using a proteomic approach. Proteomics Clin Appl. 2014;8(11-12):916-23. doi: $10.1002 /$ prca. 201300133 .

32. Moriel DG, Beatson SA, Wurpel DJ, Lipman J, Nimmo GR, Paterson $\mathrm{DL}$ et al. Identification of novel vaccine candidates against multidrugresistant Acinetobacter baumannii. PloS one. 2013;8(10):e77631. doi: 10.1371/journal.pone.0077631.

33. Tong JC, Tan TW, Ranganathan S. Methods and protocols for prediction of immunogenic epitopes. Brief Bioinform. 2006;8(2):96-108. doi: 10.1093/bib/bbl038.

34. Ni Z, Chen Y, Ong E, He Y. Antibiotic resistance determinant-focused Acinetobacter baumannii vaccine designed using reverse vaccinology. Int $\mathrm{J}$ Mol Sci. 2017;18(2):458. doi: 10.3390/ijms18020458.

35. Kim SW, Choi CH, Moon DC, Jin JS, Lee JH, Shin J-H et al. Serum resistance of Acinetobacter baumannii through the binding of factor $\mathrm{H}$ to outer membrane proteins. FEMS Microbiol Lett. 2009;301(2):224-31. doi: 10.1111/j.1574-6968.2009.01820.x

36. Jin JS, Kwon S-O, Moon DC, Gurung M, Lee JH, Kim SI et al. Acinetobacter baumannii secretes cytotoxic outer membrane protein A via outer membrane vesicles. PLoS One. 2011;6(2):e17027. doi: 10.1371/journal.pone.0017027.

37. Eijkelkamp BA, Stroeher UH, Hassan KA, Paulsen IT, Brown MH. Comparative analysis of surface-exposed virulence factors of Acinetobacter baumannii. BMC genomics. 2014;15(1):1020. doi:10.1186/1471-2164-151020 . 